\title{
IDENTIFICATION OF NUCLEASE-POSITIVE STAPHYLOCOCCI ISOLATED FROM ANIMALS
}

\author{
R. Gudding AND E. Ness \\ National Veterinary Institute, Oslo and Department of Microbiology and Immunology, \\ Norwegian College of Veterinary Medicine, Oslo, Norway
}

Summary. The nuclease-neutralisation test was evaluated as a means of identifying nuclease-positive staphylococci isolated from different animals. The test identified 510 of 520 strains $(98 \%)$ of staphylococci characterised by biochemical testing.

\section{INTRODUCTION}

The coagulase and nuclease tests are convenient and reliable methods for routine identification of human strains of Staphylococcus aureus. However, amongst staphylococci isolated from animals, two further species, $S$. intermedius and $S$. hyicus ss. hyicus, may give positive results in these tests.

Procedures for identification of $S$. aureus based on serological examinations of the nuclease have been described (Lachica et al., 1979; Gudding, 1983; Chomarat et al., 1984). The nucleaseneutralisation test distinguished $S$. aureus, $S$. intermedius, $S$. hyicus ss. hyicus and other coagulase-negative staphylococci (Gudding, 1983). This test is more simple and rapid than other methods for the identification of staphylococci (Hajek, 1976; Devriese et al., 1978; Baird-Parker, 1979). The aim of the present study was to compare standard procedures with the nucleaseneutralisation test, for identification of staphylococci isolated from different species of animals.

\section{MATERIALS AND METHODS}

Bacterial strains. A total of 520 clinical isolates of nuclease-positive staphylococci from the following animals were included: cow (mastitis), sheep (mastitis), goat (mastitis), pig (skin lesions), horse (skin lesions), dog (external otitis, skin lesions, vagina, faeces), cat (skin lesions) and poultry (septicaemia, endocarditis). All isolates produced one or more of the following enzymes: coagulase, nuclease and $a$ - and $b$-haemolysin.

Methods for identification. The organisms were identified to species level by the tubecoagulase test with rabbit plasma, the nuclease test, acetoin production, and acid production from glucose, sucrose, maltose, trehalose (aerobically) and mannitol (aerobically and anaerobically) (Baird-Parker, 1979). Nuclease production in broth cultures of staphylococci was tested in 10-mm wells in Toluidine Blue DNA Agar (TDA) (Lachica et al., 1971). Strains that produced a pink zone around the well were considered to be nuclease-positive. The production of $\alpha$ - and $\beta$ haemolysin was examined by growing the strains on bovine-blood agar.

The isolates were also subjected to a nuclease-neutralisation test (Gudding, 1983). Strains were grown in nutrient broth (Difco), and neutralisation of nuclease activity was examined in TDA Agar by transferring the broth culture to wells in the agar containing antiserum to the nucleases of $S$. aureus, $S$. intermedius or $S$. hyicus ss. hyicus. The antisera were produced in rabbits (Gudding, 1979). 


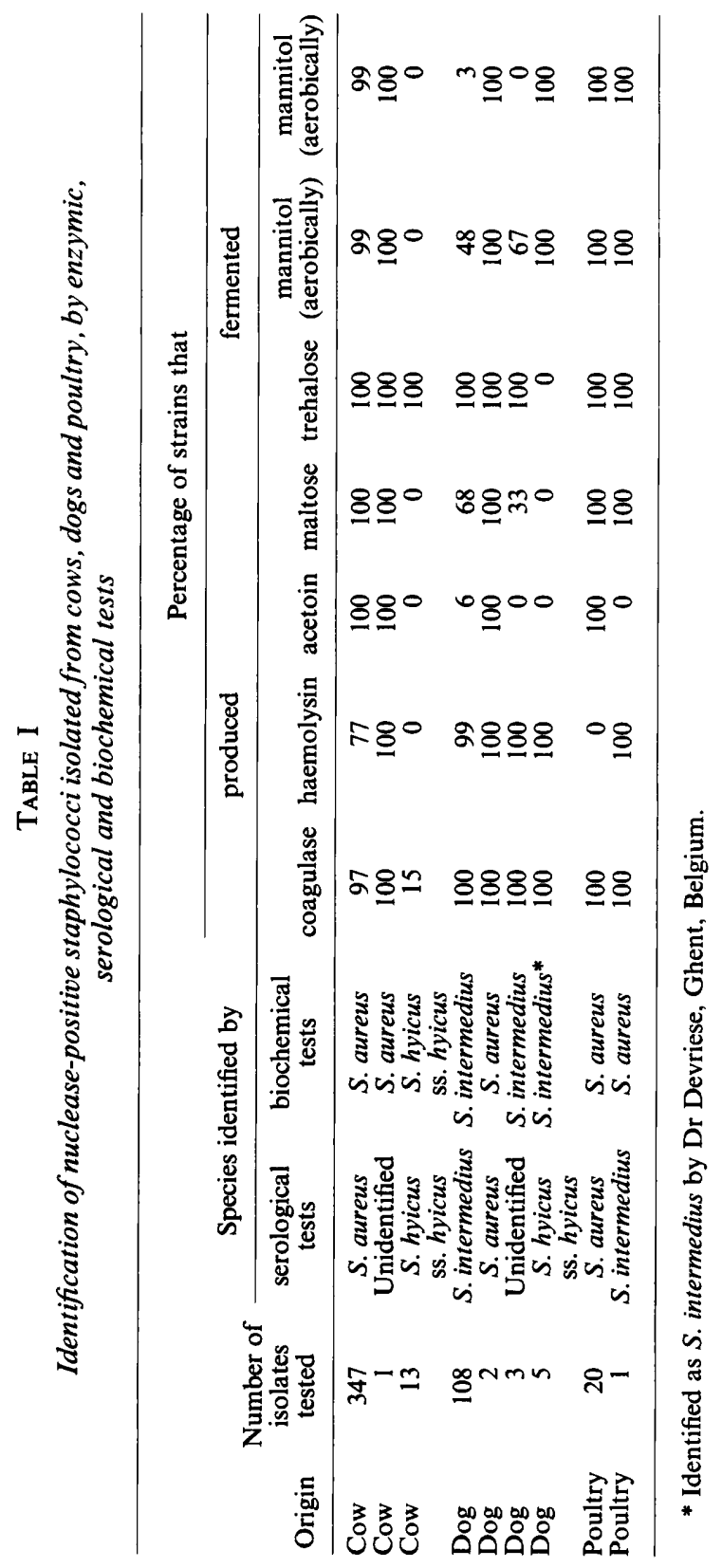




\section{RESULTS}

Bovine strains. The strains, with one exception, identified as the same species by biochemical and serological tests (table I). The nuclease of the single strain which could not be identified by the nuclease-neutralisation test was not inhibited by any of the available antisera against staphylococcal nucleases. Overall, $23 \%$ of the strains were non-haemolytic on bovine-blood agar and $3 \%$ were coagulase-negative. No strains of $S$. aureus gave negative results in both tests. With the exception of two strains which were coagulase-positive, strains of $S$. hyicus ss. hyicus isolated from mastitic milk gave identical reactions (table I).

Canine strains. Of the staphylococci isolated from dogs, $93 \%$ were identified as $S$. intermedius by the biochemical and the serological tests (table I). The strains varied in their ability to hydrolyse maltose and mannitol aerobically. A few strains of $S$. intermedius produced acetoin or hydrolysed mannitol anaerobically. Two of the strains of $S$. intermedius and both strains of $S$. aureus produced mainly $\alpha$-haemolysin. Five canine strains were inhibited by antisera to the nuclease of $S$. hyicus ss. hyicus. All these strains produced $\beta$-haemolysin and the biochemical pattern was uniform, being characterised by negative maltose and trehalose, and positive mannitol reactions. The strains were identified as $S$. intermedius by Dr L. A. Devriese, Ghent, Belgium with a more comprehensive identification scheme.

Poultry strains. Twenty of the poultry strains were identified biochemically and serologically as $S$. aureus. The nuclease of one poultry strain was inhibited by antibodies to the nuclease of $S$. intermedius. As shown in table $\mathrm{I}$, this particular strain differed from other poultry strains by being $\beta$-haemolytic and acetoin-negative.

Identification results for the 20 isolates from pigs, sheep, horses, cats and goats are summarised in table II. Biochemical and serological test results were consistent for all these isolates.

TABLE II

Distribution of species of nuclease-positive strains of staphylococci isolated from different animals

\begin{tabular}{lccc}
\hline & \multicolumn{2}{c}{ Number of isolates from each animal species } \\
\cline { 2 - 4 } Origin & S. aureus & S. intermedius & S. hyicus ss. hyicus \\
\hline Pig & 2 & 0 & 4 \\
Sheep & 6 & 0 & 0 \\
Horse & 1 & 3 & 0 \\
Cat & 2 & 1 & 0 \\
Goat & 1 & 0 & 0 \\
\hline
\end{tabular}

\section{Discussion}

The present study confirms that the serological differences between the nucleases of $S$. aureus, $S$. intermedius and $S$. hyicus ss. hyicus differentiate strains of these organisms isolated from animals. The nuclease-neutralisation test proved to be simple to perform and reliable, in that $98 \%$ of the isolates were correctly identified.

As seen from table I, S. aureus is the predominant nuclease-positive species of Staphylococcus isolated from bovine mastitis. However, $23 \%$ of these isolates were non-haemolytic when grown on bovine-blood agar and a few isolates even gave negative results in the tube coagulase test. These results, and the detection of a few strains of coagulase-positive $S$. hyicus ss. hyicus, indicate that additional tests should be used for accurate identification of staphylococci from mastitis. In the present work, all except one of the isolates of bovine $S$. aureus could be identified by the nuclease-neutralisation test, compared with $93.9 \%$ with the API Staph-Ident system (Langlois $e t$ al., 1983).

$S$. intermedius was not identified amongst the 361 isolates from bovine mastitis samples. This finding is in accordance with results of Langlois et al. $(1983,1984)$ and Biberstein et al. (1984). Watts et al. (1984) identified $S$. intermedius amongst mastitis isolates with a simplified scheme. In the present study three bovine strains of $S$. aureus might have been mis-identified as $S$. intermedius by the criteria of the latter workers. 
The great majority of canine isolates were identified as $S$. intermedius by the serological and biochemical tests. The results with canine isolates confirm data from recent American studies that c. $95 \%$ of coagulase-positive staphylococci from dogs are $S$. intermedius (Berg et al., 1984; Bieberstein et al., 1984; Cox et al., 1984). Two strains of $S$. aureus from dogs in the present study produced $\alpha$-haemolysin, a factor indicating they were of human origin. Certain characteristics such as the production of $\beta$-haemolysin and the acetoin and mannitol reactions might be used to distinguish canine isolates of the two species. However, results from the present study indicate variations in all these characters. Final identification should, therefore, be based on additional tests such as the nuclease-neutralisation test.

Five canine isolates were identified as atypical strains of $S$. intermedius by the biochemical tests and as $S$. hyicus ss. hyicus by the serological test (table I). A corresponding discrepancy was found with regard to one poultry strain. These observations emphasise the complexity of staphylococcal taxonomy. The transfer of genetic material from strains of one species of Staphylococcus to another might provide a possible explanation. Notwithstanding these few aberrant results, we conclude that the differentiation of nuclease-positive staphylococci such as $S$. aureus, $S$. intermedius and $S$. hyicus ss. hyicus can be based on the serological properties of their nucleases.

The identification of the atypical $S$. intermedius strains by Dr L. A. Devriese, Ghent, Belgium is highly appreciated.

\section{REFERENCES}

Baird-Parker A C 1979 Methods for identifying staphylococci and micrococci. In: Skinner F A, Lovelock D W (eds) Identification methods for microbiologists 2nd edn. Academic Press, London, $\mathrm{p} 201$.

Berg J N, Wendell D E, Vogelweid C, Fales W H 1984 Identification of the major coagulasepositive Staphylococcus sp. of dogs as Staphylococcus intermedius. American Journal of Veterinary Research 45: 1307-1309.

Biberstein E L, Jang S S, Hirsh D C 1984 Species distribution of coagulase-positive staphylococci in animals. Journal of Clinical Microbiology 19:610-615.

Chomarat M, Flandrois J P, Piegay M J 1984 Identification of Staphylococcus aureus nuclease by seroinhibition in blood culture supernatant fluid. Current Microbiology 11:55-58.

Cox H U, Newman S S, Roy A F, Hoskins J D 1984 Species of Staphylococcus isolated from animal infections. Cornell Veterinarian 74:124-135.

Devriese L A, Hajek V, Oeding P, Meyer S A, Schleifer K H 1978 Staphylococcus hyicus (Sompolinsky 1953) comb. var. and Staphylococcus hyicus subsp. chromogenes subsp. nov. International Journal of Systematic Bacteriology 28:482-490.

Gudding R 1979 The demonstration and characterization of deoxyribonucleases of streptococci group A, B, C, G and L. Acta Veterinaria Scandinavica 20:102-121.

Gudding R 1983 Differentiation of staphylococci on the basis of nuclease properties. Journal of Clinical Microbialogy 18: 1098-1 101.

Hajek V 1976 Staphylococcus intermedius, a new species isolated from animals. International Journal of Systematic Bacteriology 26:401-408.

Lachica R V F, Genigeorgis C, Hoeprich P D 1971 Metachromatic agar-diffusion methods for detecting staphylococcal nuclease activity. Applied Microbiology 21:585-587.

Lachica R V F, Jang S S, Hoeprich P D 1979 Thermonuclease seroinhibition test for distinguishing Staphylococcus aureus from other coagulase-positive staphylococci. Journal of Clinical Microbiology 9:141-143.

Langlois B E, Harmon R J, Akers K 1983 Identification of Staphylococcus species of bovine origin with the API Staph-Ident system. Journal of Clinical Microbiology 18:1212-1219.

Langlois B E, Harmon R J, Akers K 1984 Identification of Staphylococcus species of bovine origin with the DMS Staph-Trac system. Journal of Clinical Microbiology 20:227-230.

Watts J L, Pankey J W, Nickerson S C 1984 Evaluation of the Staph-Ident and STAPHase systems for identification of staphylococci from bovine intramammary infections. Journal of Clinical Microbiology 20:448-452. 